\title{
The CB index predicts prognosis of critically ill COVID-19 patients
}

\author{
Liang Cao ${ }^{1 \#}$, Sha Zhang ${ }^{2 \#}$, Enxin Wang ${ }^{3 \#}$, Yongchao Zhang ${ }^{3 \#}$, Yang Bai ${ }^{4}, \mathrm{Xi} \mathrm{Luo}^{5}, \mathrm{Zhe}^{6}{ }^{6}, \mathrm{Feng} \mathrm{Li}^{1}$, \\ Ling Tao $^{7,8}$, Haitao Liu ${ }^{7,8}$
}

${ }^{1}$ Department of Traditional Chinese Medicine, Xijing Hospital, Fourth Military Medical University, Xi'an, China; ${ }^{2}$ Department of Basic Medicine, Shaanxi University of Chinese Medicine, Xianyang, China; ${ }^{3}$ Department of Medical Affairs, Air Force Hospital of Western Theater Command, Chengdu, China; ${ }^{4}$ Department of Neurosurgery, General Hospital of Northern Theater Command (General Hospital of Shenyang Military Commend), Shenyang, China; ${ }^{5}$ Women's Health Centre, Xi'an People's Hospital (Xi'an Fourth Hospital), Xi'an, China; ${ }^{6}$ Department of First Clinical Medicine, Affiliated Hospital of Shaanxi University of Chinese Medicine, Xianyang, China; ${ }^{7}$ Department of Cardiology, Xijing Hospital, Fourth Military Medical University, Xi'an, China; ${ }^{8}$ Department of Infectious Disease, Hubei Women \& Children Healthcare Hospital (Guanggu District), Wuhan, China

Contributions: (I) Conception and design: L Cao, S Zhang, E Wang, F Li, L Tao, H Liu; (II) Administrative support: Z Li, L Tao, H Liu, X Luo, F Li; (III) Provision of study materials or patients: L Cao, S Zhang, Y Zhang, X Luo, E Wang, Y Bai, H Liu; (IV) Collection and assembly of data: L Cao, S Zhang, Y Zhang, X Luo, E Wang, Y Bai; (V) Data analysis and interpretation: L Cao, S Zhang, X Luo, Z Wang, Z Li, L Tao, H Liu; (VI) Manuscript writing: All authors; (VII) Final approval of manuscript: All authors.

\#These authors contributed equally to this work.

Correspondence to: Haitao Liu. Department of Cardiology, Xijing Hospital, Fourth Military Medical University, Xi’an, China. Email: cardio@fmmu.edu.cn; Ling Tao. Department of Cardiology, Xijing Hospital, Fourth Military Medical University, Xi’an, China. Email: xjcardio@hotmail.com; Feng Li. Department of Traditional Chinese Medicine, Xijing Hospital, Fourth Military Medical Universit, Xi'an, China. Email: fenglitcm@163.com.

Background: The global outbreak of COVID-19 is a significant threat to public health. Among COVID-19 cases, critically ill patients account for most in-hospital deaths. Given the pressing clinical needs, identification of potential prognostic factors that would assist clinicians to determine appropriate therapeutic interventions is urgently needed.

Methods: A retrospective analysis of 171 critically ill COVID-19 patients from two medical centers in Wuhan was conducted. The training and validation cohorts were comprised of 77 and 94 patients, respectively. Univariate and multivariate Logistic regression analyses were used to identify independent prognostic factors, and the linear prediction index was established and externally validated.

Results: Blood urine nitrogen (BUN) and high-sensitive C-reactive protein (hs-CRP) were independent factors negatively correlated with patient survival in the training cohort. A linear prediction model, named as the CB index (hs-CRP combined with BUN), was established and logistic regression analysis showed that this was associated with a $13 \%$ increase in death rate, with high sensitivity (86.7\%) and specificity (89.7\%). Patients were then divided into a high-risk group (CB index >32) and low-risk group (CB index <32) and the high-risk group showed a 56.3-fold risk of death compared with the low-risk group. Importantly, these findings were readily recaptured in the validation cohort. The efficacy of the CB index in predicting prognosis in real-world patients was then determined, which showed that patients with a higher CB index had an increased risk of death in comparison to those with a lower CB index.

Conclusions: The CB index may be an important prognostic factor in critically ill COVID-19 patients.

Keywords: Severe acute respiratory syndrome coronavirus-2 (SARS-CoV-2); coronavirus disease 2019 (COVID-19); prognostic factor; blood urine nitrogen (BUN); high-sensitive C-reactive protein

Submitted Oct 16, 2020. Accepted for publication Dec 01, 2020.

doi: $10.21037 /$ atm-20-7447

View this article at: http://dx.doi.org/10.21037/atm-20-7447 


\section{Introduction}

The outbreak of severe acute respiratory syndrome coronavirus-2 (SARS-CoV-2) and coronavirus disease 2019 (COVID-19) is a once-in-century threat to public health (1). Several concerns about the long-term challenges posed by this threat have been raised. Firstly, COVID-19 can cause death in healthy adults as well as elderly people with existing comorbidities. Although overall mortality is approximately $1 \%$, the absolute number of fatality cases is over one million. Secondly, COVID-19 transmits from person-to-person with incredible efficiency. There is strong evidence that transmission can occur in those with few symptoms and even at the pre-symptomatic stage (2-5). Indeed, COVID-19 has already caused 10 times more cases than SARS in a quarter of the time (6).

Whilst the symptoms of COVID-19 may sometimes be mild, most deaths occur in critically ill patients who have developed multiple organ dysfunction and require admission to an intensive care unit (ICU). Angiotensinconverting enzyme 2 (ACE2) is a receptor for SARS-CoV-2 that mediates virus recognition and entry to host cells (7). Histological studies have shown that ACE2 is expressed in the lung, brain, heart, liver, kidney, and intestine of COVID-19 patients and several preliminary autopsy examinations suggest that virus particles can be isolated from these organs (8). In addition to acute respiratory failure, then, critically ill patients may also present with, encephalitis, acute kidney injury, diarrhea, and an increase in myocardium enzymes and aminotransferase (9-12). This is likely because SARS-CoV-2 infection triggers a systemic immune response, activating immune cells to attack ACE2expressing cells, leading to both virus clearance and multiple organ damage.

Despite recent progress in the development of specific therapies to treat COVID-19 such as vaccines and convalescent plasma $(13,14)$, the prognosis of critically ill patients remains poor and there is an urgent need to establish biomarkers that assist in predicting therapeutic outcomes and prognosis. We present the following article in accordance with the STROBE reporting checklist (available at http://dx.doi.org/10.21037/atm-20-7447).

\section{Methods}

\section{Study design and patient enrollment}

This retrospective, observational study aimed to identify prognostic biomarkers for critically ill COVID-19 patients who, following the New Coronavirus Pneumonia Diagnosis and Treatment Program ( $6^{\text {th }}$ edition), were defined as those admitted to ICU and requiring mechanical ventilation. Two cohorts of laboratory confirmed, and critically ill COVID-19 patients were enrolled. Training cohort study cases were obtained from a consecutive historical cohort of 77 patients treated in the ICU of the Hubei Women \& Children Healthcare Hospital (Guanggu Hospital District) between February $19^{\text {th }}$ and April $17^{\text {th }}$ in 2020. The validation cohort consisted of 94 consecutive patients from the ICU of the Wuhan Huoshenshan Hospital between February $8^{\text {th }}$ and April $6^{\text {th }}$. Both hospitals are specifically designated to treat COVID-19 patients. All patients were laboratory confirmed by SARS-CoV-2 real-time PCR assay and were followed-up until discharged with recovery, or death. All procedures performed in this study involving human participants were in accordance with the Declaration of Helsinki (as revised in 2013). The study was approved by the Medical Ethics Committees of the Hubei Women \& Children Healthcare Hospital (No. 2020-FYGG-011). Written informed consent was obtained from each participant.

\section{Data collection}

The electronic medical records, nursing records, and laboratory examinations of each patient were reviewed by at least two independent, experienced medical doctors. Admission data were recorded following the International Severe Acute Respiratory and Emerging Infection Consortium case report forms. The following data were collected: age, sex, exposure history, chronic comorbidities (chronic cardiac disease, chronic pulmonary disease, cerebrovascular disease, diabetes), symptoms from the onset of disease (fever, cough, shortness of breath, myalgia, diarrhea), and laboratory findings at admission (white blood cell count, lymphocyte count, hemoglobin concentration, platelet count, CRP level, aminotransferase concentration, BUN concentration, and creatinine concentration).

\section{Statistical analysis}

Categorical variables were described as the frequency and percentage, and continuous variables as the median with interquartile range (IQR). The primary endpoint was survival during patient admission. A univariate or multivariate logistic regression model was adopted to analyze the prognostic factors of critically ill patients. 
Table 1 Baseline characteristics of the enrolled critically ill COVID-19 patients

\begin{tabular}{|c|c|c|c|}
\hline Characteristics & Training group $(n=77)$ & Verification group $(n=94)$ & $P$ value \\
\hline WBC $\left(10^{9} / \mathrm{L}\right)$ & $5.4(3.9-8.3)$ & $6.4(5.0-9.2)$ & 0.471 \\
\hline Neutrophil $\left(10^{9} / \mathrm{L}\right)$ & $4.4(2.6-7.0)$ & $4.4(3.0-7.9)$ & 0.555 \\
\hline Lymphocyte ( $\left.10^{9} / \mathrm{L}\right)$ & $1.1(0.7-1.6)$ & $1.1(0.7-1.5)$ & 0.071 \\
\hline Monocyte $\left(10^{9} / \mathrm{L}\right)$ & $0.4(0.3-0.4)$ & $0.38(0.27-0.50)$ & 0.396 \\
\hline ALT (U/L) & $18.9(13.5-28.3)$ & $23.7(16.3-43.7)$ & 0.146 \\
\hline AST (U/L) & $23.6(15.5-30.7)$ & $25.3(18.3-36.5)$ & 0.424 \\
\hline Total bilirubin ( $\mu \mathrm{mol} / \mathrm{L})$ & $11.0(7.0-14.7)$ & $10.3(8.4-14.0)$ & 0.244 \\
\hline Albumin (g/L) & $34.5(29.0-38.7)$ & $33.8(30.5-37.6)$ & 0.437 \\
\hline hs-CRP (mg/L) & $9.1(2.3-84.7)$ & $14.7(2.5-103.5)$ & 0.379 \\
\hline
\end{tabular}

WBC, white blood cell; ALT, alanine aminotransferase; AST, aspartate aminotransferase; BUN, blood urea nitrogen; hs-CRP, high sensitive $C$ reaction protein; IQR, interquartile range.

Based on the independent prognostic factors, a scoring system for predicting survival was proposed and the area under the receiver operating characteristic (ROC) curve was used to evaluate its predictive ability. Cut-off values were determined according to the ROC curves and models with an area under the ROC $>0.7$ generally considered to be useful and predictive. To validate the previous findings, similar analyses were repeated in the verification cohort. Statistical analysis was performed using SPSS for Windows, version 17.0 , and a two-sided $\mathrm{P}$ value $<0.05$ was considered significant.

\section{Results}

\section{Patient characteristics}

A total of 171 critically ill COVID-19 patients were enrolled with 77 patients placed in the training cohort and 94 in the validation cohort. Detailed patient characteristics are shown in Table 1. The two cohorts were well balanced, with comparable levels of age, sex, chronic comorbidities and clinical manifestations (data not shown). The blood cell count and biochemistry data of patient's was analyzed at admission. As shown in Table 1, there was no significant difference in white blood cell (WBC), neutrophil, lymphocyte, monocyte, and platelet count between the two groups. Biochemistry analysis including alanine aminotransferase (ALT), aspartate aminotransferase (AST), total bilirubin (TBIL), albumin (ALB), blood urea nitrogen (BUN), and serum creatinine were mostly within the normal range, and comparable between the two groups. It was noted that 13 patients in the training cohort and 22 in the validation cohort had higher levels of high-sensitive C-reactive protein (hs-CRP), whereas the median values of hs-CRP in the two groups were comparable (Table 1).

\section{Identification of prognostic factors for critically ill COVID-19}

Results of the univariate logistic regression analysis show that AST ( $R R=1.08, \mathrm{P}=0.006)$, TBIL $(\mathrm{RR}=1.14, \mathrm{P}=0.023)$, BUN ( $R R=1.38, P=0.002)$, serum creatinine $(R R=1.03, P=0.012)$, and hs-CRP $(\mathrm{RR}=1.03, \mathrm{P}<0.001)$ were important correlation factors for prognosis, whereas ALB $(\mathrm{RR}=0.86, \mathrm{P}<0.001)$ was not (Table 2). Importantly, an increase in findings with correlation indicated an increased risk of disease-associated death. The results also showed that the neutrophil/ lymphocyte ratio (NLR, RR $=1.27, \mathrm{P}<0.001$ ) appeared to be correlated with prognosis, although a multivariate model including all significant variables identified in the univariate analysis did not yield NLR as an independent prognostic factor. Moreover, the multivariate analysis showed that BUN $(\mathrm{RR}=1.36, \mathrm{P}=0.028)$ and hs-CRP $(\mathrm{RR}=1.02, \mathrm{P}=0.006)$ were independent prognostic factors (Table 2). 
Table 2 Univariate and multivariate logistic regression analysis for prognostic factors of critically ill COVID-19 patients

\begin{tabular}{|c|c|c|c|c|}
\hline Variables & \multicolumn{2}{|c|}{ Univariate analysis } & \multicolumn{2}{|c|}{ Multivariate analyses } \\
\hline NLR & $1.27(1.12-1.45)$ & $<0.001$ & - & - \\
\hline Platelet, per $10^{9} / \mathrm{L}$ increase & $1.00(0.99-1.00)$ & 0.154 & - & - \\
\hline ALT, per $1 \mathrm{U} / \mathrm{L}$ increase & $1.02(1.00-1.04)$ & 0.128 & - & - \\
\hline Total bilirubin, per $1 \mu \mathrm{mol} / \mathrm{L}$ increase & $1.14(1.02-1.27)$ & 0.023 & - & - \\
\hline Albumin, per $1 \mathrm{~g} / \mathrm{L}$ increase & $0.82(0.74-0.92)$ & $<0.001$ & - & - \\
\hline BUN, per $1 \mathrm{mmol} / \mathrm{L}$ increase & $1.38(1.13-1.68)$ & 0.002 & $1.36(1.03-1.80)$ & 0.028 \\
\hline Serum creatinine, per $1 \mu \mathrm{mol} / \mathrm{L}$ increase & $1.03(1.01-1.05)$ & 0.012 & - & - \\
\hline
\end{tabular}

NLR, neutrophil/lymphocyte ratio; ALT, alanine aminotransferase; AST, aspartate aminotransferase; BUN, blood urea nitrogen; hs-CRP, high sensitive $C$ reaction protein; IQR, interquartile range.

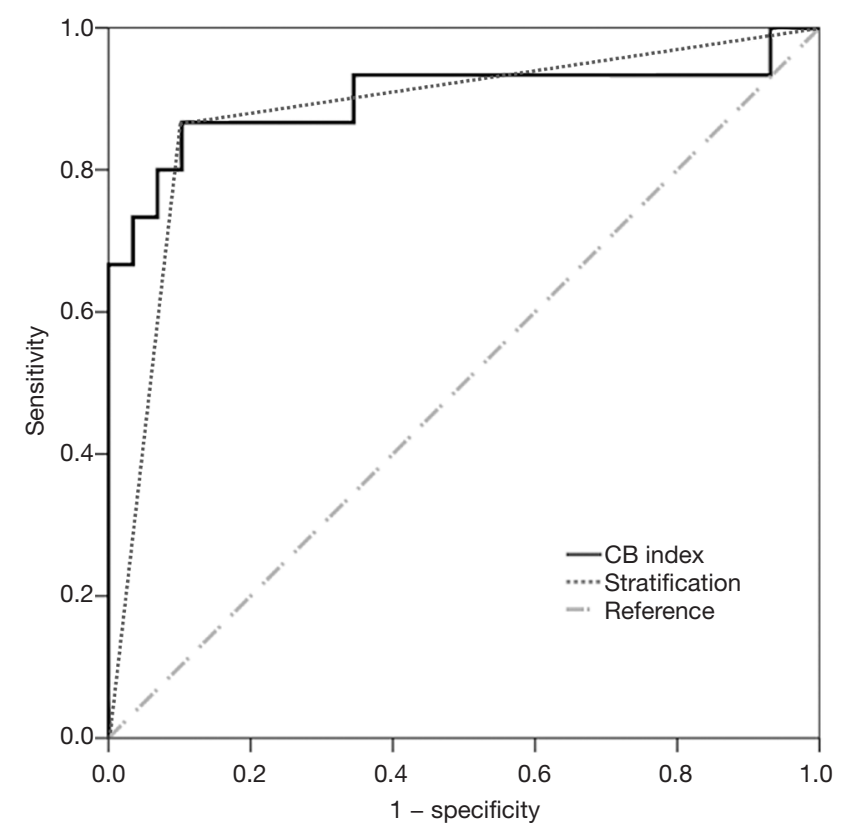

Figure 1 Receiver operating characteristic (ROC) curve of the CB index in the training cohort. The area under the curve (AUC) of $\mathrm{CB}$ index was 0.901 .

\section{Establishment of the prognostic index}

Having established that hs-CRP and BUN were two independent prognostic factors for critically ill COVID-19 patients, their sensitivity and specificity in predicting prognosis were then determined. Regression coefficients were first calculated, showing hs-CRP $(\ln =0.020)$ and BUN $(\ln =0.307)$. The linear predicting index including the two factors was termed as the CB index and established by the following equation: linear Prediction $(\mathrm{LP})=0.2 \times$ hs-CRP $+3.1 \times$ BUN. Logistic regression analysis showed that the $\mathrm{CB}$ index elicited a predicting value for patient survival (RR $=1.13,95 \%$ CI: $1.06-1.20, \mathrm{P}<0.001$ ), in which a one-unit increase would add mortality risk by $13 \%$. The area under the ROC curve was as high as 0.901 (95\% CI: $0.78-1.00$ ). The maximum value of the Youden index in the ROC curve was 0.764 , which was set as the cut-off value, and the value of the corresponding $\mathrm{CB}$ index in predicting prognosis was 32.295 (Figure 1). The training cohort was stratified according to this cut-off value. Of the 44 available patients, those with a CB index higher than 32 were designated as a high-risk group (17 cases) while those whose index was lower than 32 were designated as a low-risk group (27 cases). In comparison with the low-risk group, the highrisk group had a markedly increased risk of death, yielded with a RR of 56.3 (95\% CI: 8.35-380.06).

\section{Validation of the CB index as a prognostic factor}

These results highlighted the CB index as a favorable prognostic factor for critically ill COVID-19 patients. To test whether this index universally correlated with patient survival, we validated it in another cohort, consisting of 94 patients from the ICU of the Wuhan Huoshenshan Hospital. The ROC curve was also used to measure the prediction capacity of the $\mathrm{CB}$ index in the verification 


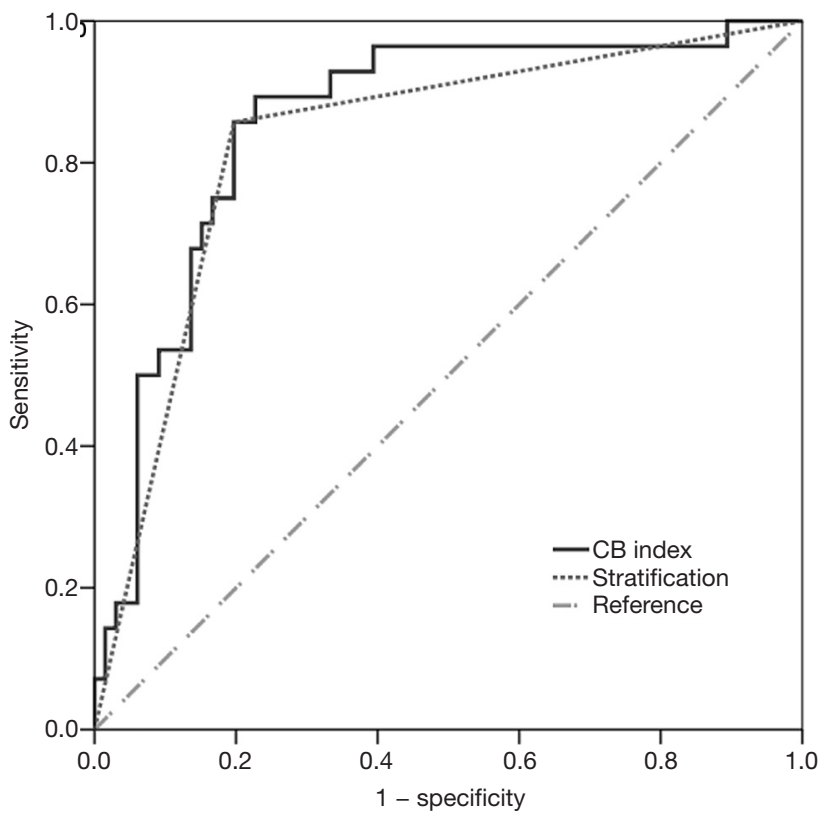

Figure 2 Receiver operating characteristic (ROC) curve of the $\mathrm{CB}$ index in the verification cohort. The area under the curve (AUC) of CB index was 0.857 .

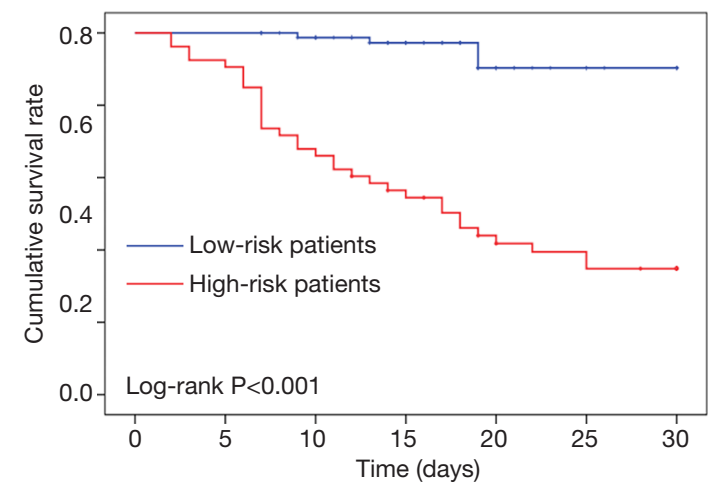

Figure 3 Kaplan-Meier plots showing the cumulative survival rate of critically ill COVID-19 patients who were stratified into two groups according to the $\mathrm{CB}$ index. Blue line, $\mathrm{CB}$ index $<32, \mathrm{n}=85$; Red line, CB index $>32, \mathrm{n}=53$; log-rank test for trend, $\mathrm{P}<0.001$.

cohort. The area under the ROC curve was 0.857 (95\% CI: 0.77-0.94). The maximum value of the Youden index in the ROC curve was set as the cut-off value, and the corresponding CB index was 33.238 , with sensitivity of $82.1 \%$ and specificity of $80.3 \%$. These data were highly consistent with those in the training cohort, suggesting that the CB index universally indicated prognosis and yielded consistent results (Figure 2). After stratification at the cutoff value for the CB index, 37 patients were allocated to a high-risk group and 57 to a low-risk group. Logistic regression analysis showed that the high-risk group had a 24.5 -fold (95\% CI: 7.22-82.86) increased risk of death compared with the low-risk group. These findings further confirmed that the CB index negatively correlated with the prognosis of critically ill COVID-19 patients.

\section{Performance of CB index in real-world patients}

The CB index in real-world critically ill COVID-19 patients was then assessed. The survival information of 44 available patients from the training cohort and 94 from the verification cohort was analyzed. Of the 138 patients, 85 were in the low-risk group (CB index $<32)$ and 53 were in the high-risk group ( $\mathrm{CB}$ index $>32)$. The primary end point was overall survival during hospitalization. As shown in Figure 3, the Kaplan-Meier survival curve indicated that patients in the high-risk group had a significantly higher risk of death than those in the low-risk group. At the end of follow up, $7 \%$ of patients in the low-risk group had died in comparison to $70 \%$ of those in the high-risk group.

\section{Discussion}

Despite a world-wide concerted effort, the treatment of critically ill COVID-19 patients remains largely supportive and its efficacy is far from satisfactory. The overall death rate of COVID-19 is around $1 \%$, however, patients with mild disease may not seek medical attention, the case fatality rate in real-world would be less than $1 \%$ (2). In contrast, critically ill patients usually require more aggressive treatments to relieve symptoms and reduce mortality. Previous studies have suggested that the 28-day mortality of critically ill COVID-19 patients is approximately 50\% (4). In these circumstances, the identification of prognostic factors would significantly assist clinicians to determine a tailored therapeutic strategy designed to reduce mortality.

A panel of studies revealed several risk factors which are associated with poor prognosis in COVID-19 patients, including pre-existing chronic pulmonary disease, older age, lymphocytopenia, a high SOFA score, elevated levels of LDH, and myocardium enzymes $(4,8)$. However, these studies did not focus on disease severity on admission. To this end, our study focused on critically ill patients during the peak of the COVID-19 impact in Wuhan. One recent study showed that NLR is an independent risk factor 
for critically ill patients, with a 15.04-fold higher risk of death (15). Our univariate analysis found that whilst NLR seemed to correlate with prognosis, the multivariate analysis subjected to the parameters did not yield NLR as an independent prognostic factor. This paradox might be attributed to patient heterogeneity between different medical centers, indicating that potential prognostic factors need to be verified in different medical centers.

Our findings provide important parameters, including hs$\mathrm{CRP}$ and BUN, for further analysis. It is not surprising that hs-CRP is an independent prognostic factor because critically ill COVID-19 patients usually elicit an inflammatory cytokine storm $(16,17)$. Moreover, previous studies have reported that the serum concentration of hs-CRP in symptomatic patients is much higher than that found in asymptomatic patients $(4,18)$. These results prompted us to recognize hs$\mathrm{CRP}$ as an independent prognostic factor. Given that ACE2, the cell entry gate for SARS-CoV-2, is universally expressed in the lung, brain, heart, liver, kidney, and intestine, it is unsurprising to see that SARS-CoV-2 infection results in damage to multiple organs (9-12). We found that while BUN is also a prognostic factor for critically ill COVID-19 patients, AST, total bilirubin, ALB, and serum creatinine are not. A possible explanation for this is that the kidney is the most vulnerable organ in response to viremia and cytokine storm, and damage to it takes place prior to occurring in other organs. In contrast to serum creatinine that primarily reflects renal function, BUN concentration is also affected by protein intake and absorption (intestine function), protein synthesis and metabolism (liver function). This suggests BUN is superior to serum creatinine in reflecting multiple organ function $(19,20)$.

To make hs-CRP and BUN more reliable as prognostic factors, we established a linear predicting index (CB index) that combines inflammation (hs-CRP) with organ function (BUN). This novel index not only reflects the severity of SARS-CoV-2 infection, but also mirrors secondary organ damage. We found the CB index correlated with the risk of death with a RR of 1.13 , and that after stratification at the cut-off value of 32 , the index universally possessed high predicting significance in both training and verification cohorts, with a RR of 56.3 and 24.5 , respectively. This finding may have pivotal clinical significance, by indicating that patients with a CB index higher than 32 have an increased risk of death and should be treated with more aggressive interventions than those with a lower index. Since this study was undertaken, we have divided critically ill COVID-19 patients in clinical practice into high and low risk groups according to their corresponding CB index values. Patients in high-risk groups have been treated with more aggressive interventions, including mechanical ventilation, tocilizumab, enteral and parenteral nutrition support, and continuous renal replacement therapy. This has dramatically changed therapeutic outcomes of patients and reduced the overall mortality to approximately $4 \%$ (unpublished data). As the global outbreak of COVID-19 continues, we believe that the CB index will help more clinicians to determine therapeutic approaches for critically ill patients.

This study has several limitations. Some cases had incomplete laboratory test documentation whilst others were diagnosed elsewhere and had incomplete medical information. Moreover, as all enrolled cases were from Wuhan, the application of these findings to other locations is uncertain. Finally, many patients did not undergo sputum bacterial and fungal assessment on admission because medical resources were largely overwhelmed. We anticipate that critically ill COVID-19 patients with concurrent bacterial or fungal infection would have an even higher $\mathrm{CB}$ index and an even worse prognosis.

Taken together, this study highlighted the CB index as a reliable prognostic factor for critically ill COVID-19 patients. Calculating and monitoring the $\mathrm{CB}$ index will assist medical doctors to reduce mortality, and eventually win the war against SARS-CoV-2.

\section{Acknowledgments}

We thank our partners for their assistance during this research. We greatly appreciate all of our front-line health care colleagues in Wuhan.

Funding: The Key Project of Social Development, Shaanxi Province (\#2017ZDXM-SF-049 to HL and \#2020JQ863 to SZ); The National Natural Science Foundation of China (\#81803950 to SZ); The Project of Xi'an Science and Technology (\#2016046SF/XY05-1 to HL); The Subject Innovation Team of Shaanxi University of Chinese Medicine (\#2019-YS01 to ZL) and Xi'an Fourth Hospital Research Incubation Fund (\#FZ-35 to XL).

\section{Footnote}

Reporting Checklist: The authors have completed the STROBE reporting checklist. Available at http://dx.doi. org/10.21037/atm-20-7447

Data Sharing Statement: Available at http://dx.doi. 
org/10.21037/atm-20-7447

Conflicts of Interest: All authors have completed the ICMJE uniform disclosure form (available at http://dx.doi. org/10.21037/atm-20-7447). The authors have no conflicts of interest to declare.

Ethical Statement: The authors are accountable for all aspects of the work in ensuring that questions related to the accuracy or integrity of any part of the work are appropriately investigated and resolved. All procedures performed in this study involving human participants were in accordance with the Declaration of Helsinki (as revised in 2013). This study was approved by the Ethics Medical Committee of Hubei Women \& Children Healthcare Hospital (No.2020-FYGG-011). Written consent was obtained from each participant.

Open Access Statement: This is an Open Access article distributed in accordance with the Creative Commons Attribution-NonCommercial-NoDerivs 4.0 International License (CC BY-NC-ND 4.0), which permits the noncommercial replication and distribution of the article with the strict proviso that no changes or edits are made and the original work is properly cited (including links to both the formal publication through the relevant DOI and the license). See: https://creativecommons.org/licenses/by-nc-nd/4.0/.

\section{References}

1. Jones DS. History in a Crisis - Lessons for Covid-19. N Engl J Med 2020;382:1681-3.

2. Guan WJ, Ni ZY, Hu Y, et al. Clinical Characteristics of Coronavirus Disease 2019 in China. N Engl J Med 2020;382:1708-20.

3. Yang X, Yu Y, Xu J, et al. Clinical course and outcomes of critically ill patients with SARS-CoV-2 pneumonia in Wuhan, China: a single-centered, retrospective, observational study. Lancet Respir Med 2020;8:475-81.

4. Zhou F, Yu T, Du R, et al. Clinical course and risk factors for mortality of adult inpatients with COVID-19 in Wuhan, China: a retrospective cohort study. Lancet 2020;395:1054-62.

5. Li Q, Guan X, Wu P, et al. Early Transmission Dynamics in Wuhan, China, of Novel Coronavirus-Infected Pneumonia. N Engl J Med 2020;382:1199-207.

6. Mahase E. Coronavirus covid-19 has killed more people than SARS and MERS combined, despite lower case fatality rate. BMJ 2020;368:m641.
7. Lan J, Ge J, Yu J, et al. Structure of the SARS-CoV-2 spike receptor-binding domain bound to the ACE2 receptor. Nature 2020;581:215-20.

8. Hanley B, Lucas SB, Youd E, et al. Autopsy in suspected COVID-19 cases. J Clin Pathol 2020;73:239-42.

9. Ronco C, Reis T. Kidney involvement in COVID-19 and rationale for extracorporeal therapies. Nat Rev Nephrol 2020;16:308-10.

10. Ye M, Ren Y, Lv T. Encephalitis as a clinical manifestation of COVID-19. Brain Behav Immun 2020;88:945-946.

11. Song Y, Liu P, Shi XL, et al. SARS-CoV-2 induced diarrhoea as onset symptom in patient with COVID-19. Gut 2020;69:1143-4.

12. Zhang C, Shi L, Wang FS. Liver injury in COVID-19: management and challenges. Lancet Gastroenterol Hepatol 2020;5:428-30.

13. Ye M, Fu D, Ren Y, et al. Treatment with convalescent plasma for COVID-19 patients in Wuhan, China. J Med Virol 2020;92:1890-901.

14. Thanh Le T, Andreadakis Z, Kumar A, et al. The COVID-19 vaccine development landscape. Nat Rev Drug Discov 2020;19:305-6.

15. Liu Y, Du X, Chen J, et al. Neutrophil-to-lymphocyte ratio as an independent risk factor for mortality in hospitalized patients with COVID-19. J Infect 2020;81:e6-12.

16. Herold T, Jurinovic V, Arnreich C, et al. Elevated levels of IL-6 and CRP predict the need for mechanical ventilation in COVID-19. J Allergy Clin Immunol 2020;146:128-36.e4.

17. Chen W, Zheng KI, Liu S, et al. Plasma CRP level is positively associated with the severity of COVID-19. Ann Clin Microbiol Antimicrob 2020;19:18.

18. Liu F, Li L, Xu M, et al. Prognostic value of interleukin-6, C-reactive protein, and procalcitonin in patients with COVID-19. J Clin Virol 2020;127:104370.

19. Bingham SA. Urine nitrogen as a biomarker for the validation of dietary protein intake. J NUTR 2003;133 Suppl 3:921S-4S.

20. Di Micco L, Quinn RR, Ronksley PE, et al. Urine creatinine excretion and clinical outcomes in CKD. Clin J Am Soc Nephrol 2013;8:1877-83.

(English Language Editor: B. Draper)

Cite this article as: Cao L, Zhang S, Wang E, Zhang Y, Bai Y, Luo X, Li Z, Li F, Tao L, Liu H. The CB index predicts prognosis of critically ill COVID-19 patients. Ann Transl Med 2020;8(24):1654. doi: 10.21037/atm-20-7447 